\title{
Modelling ground vibrations induced by harmonic loads
}

1 Nawras Hamdan PhD

Geotechnical Engineer, Lloyd's Register EMEA, Aberdeen, UK (corresponding author: nawras.hamdan@|r.org)

2 Omar Laghrouche PhD, CEng, FICE, FHEA

Professor, Institute for Infrastructure and Environment, Heriot-Watt University, Edinburgh, UK
3 David Connolly $\mathrm{PhD}$

Assistant Professor, Institute for Infrastructure and Environment Heriot-Watt University, Edinburgh, UK

4 Peter K. Woodward PhD, CEng, FICE, FHEA Atkins Professor of High Speed Railways/Director of Research Institute, Institute for Infrastructure and Environment, Heriot-Watt University, Edinburgh, UK
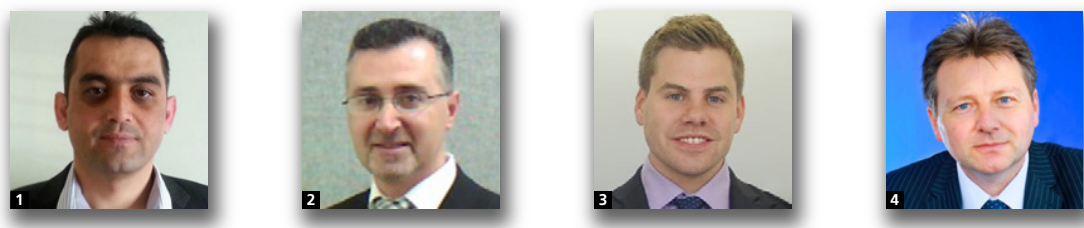

A finite-element model combining the frequency domain thin-layer method with paraxial boundary conditions to simulate the semi-infinite extent of a soil medium is presented in this paper. The combined numerical model is used to deal with harmonic vibrations of surface rigid foundations on non-horizontal soil profiles. The model can deal with soil media over rigid bedrock or significant depths of half-space. Structured finite elements are used to mesh simple geometry soil domains, whereas unstructured triangular mesh grids are employed to deal with complex geometry problems. Dynamic responses of homogeneous as well as layered soil profiles are simulated and validated against analytical and approximate solutions. Finally, the model is used to deal with surface ground vibration reduction, in which it is first validated against published results and then followed by an example involving a bridge.

\section{Notation}

A, $\mathbf{G} \quad$ matrices dependent on geometry and material properties of layered region

$A_{0}$

$A_{\mathrm{r}}$

$c_{\mathrm{p}}$

$c_{\mathrm{s}}$

D, B

$E_{\mathrm{c}}$

$E_{\mathrm{s}}$

$f_{j}^{\mathrm{v}}$

$\mathbf{K}_{\mathrm{s}}$

area under vertical displacement curve

dimensionless frequency

amplitude reduction factor

pressure wave velocity

shear wave velocity

matrices dependent on material properties of layered region

elasticity modulus of concrete

elasticity modulus of soil

natural frequencies of vertical excitation

fundamental frequency of upper layer

fundamental frequency of bottom layer

diagonal matrix containing wavenumber

thickness of soil domain

thickness of upper soil layer

thickness of bottom soil layer

imaginary number

approximate stiffness matrix of SV-P waves in a

half-space

stiffness matrix of the domain

wavenumber

dynamic stiffness matrix of consistent transmitting

boundary on left side
M

$\mathbf{M}_{\mathrm{s}}$

$n$

$\boldsymbol{P}$

$\boldsymbol{P}_{\mathrm{L}}$

$\boldsymbol{P}_{\mathrm{R}}$

$\mathbf{R}$

$U$

$U_{\mathrm{L}}$

$\boldsymbol{U}_{\mathrm{R}}$

$u_{R}$

V

$\mathbf{V}^{-1}$

X

$z_{i}$ mass matrix of layered region

mass matrix of domain

number of soil layers

nodal forces vector

nodal force vector on left side of domain

nodal force vector on right side of domain

dynamic stiffness matrix of consistent transmitting boundary on right side

nodal displacement vector

nodal displacement vector of left side vertical

boundary

nodal displacement vector of right side vertical

boundary

contains eigenvectors

modal matrix contains eigenmodes

inverse of modal matrix

distance between applied load and edge of bridge

thickness of each soil layer

rate of linearity

damping coefficient

ratio of shear wave velocity to dilatational wave velocity

rigid bedrock inclination angle/soil layer interface

inclination angle

Rayleigh wavelength

shear modulus 


$\begin{array}{ll}\mu_{0} & \text { shear modulus of surface soil layer } \\ \mu_{1} & \text { shear modulus of upper soil layer } \\ \mu_{2} & \text { shear modulus of bottom soil layer } \\ v & \text { Poisson ratio of soil } \\ \rho & \text { density of soil } \\ \rho_{1} & \text { density of upper soil layer } \\ \rho_{2} & \text { density of bottom soil layer } \\ \omega & \text { circular frequency }\end{array}$

\section{Introduction}

Dynamic response of soil media and surface rigid foundations has been investigated intensively using various numerical techniques such as the finite-element method (FEM) and the boundary-element method (BEM). The BEM became popular for such problems thanks to its ability to incorporate automatically wave radiation to infinity and hence simulate the semi-infinite extent of soil media. The FEM is more flexible in dealing with material and geometry complexities; however, for wave radiation to infinity it requires special treatment of the boundaries. Combined FEM-BEM models are very practical for such problems, in which all discontinuities are contained within the finite-element domain and the far field is modelled by way of boundary integrals.

Examples of BEM approaches include the work of Beskos et al. (1986), who presented a comprehensive review of the work carried out on the dynamics of foundations up to the 1980s. The BEM was employed by von Estroff and Schmid (1984) to model the dynamic behaviour of a strip foundation overlying a soil stratum over bedrock and by Abascal and Dominguez (1984), who used the same technique for simulating the dynamic behaviour of strip foundations over half-space. Spyrakos and Beskos (1986) developed a boundary-element time domain method and examined the dynamic response of surface and embedded foundations under plane strain conditions. Israil and Ahmad (1989) presented a parametric study using the BEM for modelling the dynamic behaviour of strip foundations over homogeneous half-space media and then over a soil deposit over either half-space or bedrock. The authors investigated the effect of material properties such as damping and relative stiffness between the soil layers. They also considered the effect of the half-space and the stratum depth. A detailed review of BEM, its formulation and applications can be found, for example, in the review paper by Liu et al. (2011) or in text books such as Boundary Element Methods for SoilStructure Interaction by Hall and Oliveto (2003). More recently, Romero et al. (2013a) used a three-dimensional (3D) non-linear BEM-FEM to deal with soil-structure interaction problems and Romero et al. (2013b) investigated the dynamic soil-bridge interaction in high-speed rail lines. The BEM was also coupled with the FEM to deal with train-induced ground vibrations for example, Costa et al. (2012) and Barbosa et al. (2015).

The FEM has also been extensively used to simulate soil dynamic problems but, given the semi-infinite extent of soil media, as indicated above, special conditions are required at the computational domain boundaries to prevent wave reflection. This has led to the development of various approaches that combine a finite-element model with local or non-local boundary condition operators. Such approaches include, for example, the FEMBEM combined model as mentioned above, finite-infinite elements such as in the paper by Yang et al. (2007), absorbing boundary layers and dampers as seen in the paper by Semblat et al. (2010), and the perfectly matched layer (PML) of Basu and Chopra (2003, 2004), Lee et al. (2014) and Lopes et al. (2014).

In the present work, the FEM is used with the thin-layer method (TLM) and paraxial boundary condition (PBC) to simulate the semi-infinite extent of soil media. The TLM was first developed by Waas (1972) and has been extended and used by various other authors such as Kausel (1974), Tassoulas (1981), Laghrouche (1996), Park and Tassoulas (2002), Ikeda and Tassoulas (2008), Hamdan et al. (2013) and others. It can be used to compute the nodal forces needed on the lateral domain boundaries to allow wave radiation to infinity. Regarding PBC, the dynamic stiffness of a half-space medium is approximated and coupled to a soil domain model to allow waves to propagate with respect to depth, with no or very little reflection. Kausel and Roësset (1981) employed the ThomsonHaskell transfer matrix technique to derive the dynamic stiffness matrix for layered media. This approach was also extended by Andrade (1999), who obtained a formulation for a halfspace element and assembled its contribution to adjacent finite elements of the soil domain. A further development was achieved by Lee et al. (2011a), who dealt with saturated halfspace media under plane strain conditions, while Lee et al. (2011b) considered porous half-space media. The TLM was combined with PBC by Hamdan et al. (2015a), where the authors investigated the effect of the thickness of a buffer layer on the performance of the combined model. It is worth noting that the TLM was also combined with the PML method by Barbosa et al. (2012), where it proved effective in terms of accuracy and computational cost. Finally, it is useful to mention that the TLM has also been used by Park and Kausel (2004) to compute dispersion curves for anti-plane and in-plane waves in unbounded domains.

Analytical and numerical solutions have been developed for computing the fundamental frequency or the dynamic compliances of surface rigid foundations overlying rigid bedrock. However, there are no such solutions or even empirical expressions for the case of inclined rigid bedrock or inclined soil layers. These cases are important from a practical point of view for investigating, for example, cases of vibratory machines sensitive to minor changes in the fundamental frequency of the underlain soil profiles. It will be shown that the proposed model can deal with such cases and that geometry complexities can be accommodated by the use of unstructured mesh grids. For the purpose of validation, the combined model is used in the case of a homogeneous half-space soil to compare with 
published results, when a sufficient thickness of the half-space is taken into account.

The outline of this paper is as follows. A brief description of the coupled finite-element model is given in Section 2. In Section 3, theoretical models providing the natural frequencies of a soil layer over a rigid base and for a two-layer soil profile are presented. The presented coupled finite-element model is then used in Section 4 to examine the effect of the inclination of both the bedrock and the soil layer interface, in the case of a two-layer soil profile, on the dynamic response of a surface rigid foundation. The effect of the relative thickness and stiffness of the soil layers is also investigated. In Section 5, the dynamic behaviour of a multi-layered soil profile is examined and in Section 6 an example of ground vibration reduction is presented. Finally, conclusions are drawn in the last section based on the numerical results.

\section{Method of analysis}

The TLM technique is based on subdividing the soil domain layers into sub-layers and employing a plane wave propagating in the horizontal direction within each sub-layer. In this work, linear variation of the displacement field is assumed in the vertical direction with the thickness of the sub-layers not exceeding one-tenth of the shear wavelength, as reported by Waas (1972). This approach is used for deriving nodal forces to be used on the vertical boundaries of the computational domain to allow wave radiation to infinity. Models based on this approach are capable of dealing with problems involving complex geometries with variations in material properties. Structured mesh grids have been used in most associated publications with this approach. However, it is usually not practical to employ such mesh grids in engineering applications where complex geometries have to be considered. Chen (1994), for example, used eight-node quadrilateral finite elements in the irregular region and, in order to guarantee the linear variation of displacement with respect to depth at the lateral boundaries, the author employed seven-node quadrilateral finite elements, which have only two nodes at the interface of the irregular and regular regions. Recently, Jones and Hunt (2011) used a stepwise fashion technique to approximate the soil layer interface inclination. Both techniques are useful to model soil media with non-horizontal profiles; however, the development of a more flexible procedure is desirable. In the current work, a practical technique is employed by meshing the irregular domain randomly using an automatic mesh generator provided by Geuzaine and Remacle (2009). The flexibility and practicality of the unstructured mesh grids have merits, as they allow practitioners to deal with complex engineering problems. A typical soil-structure interaction problem is depicted in Figure 1, in which the soil profile consists of non-horizontal soil layers resting on bedrock and including some man-made discontinuities such as tunnels, structures, foundations and wave barriers. Practical problems dealing with surface reduction of groundborne vibration are presented by Hamdan et al. (2015b), where

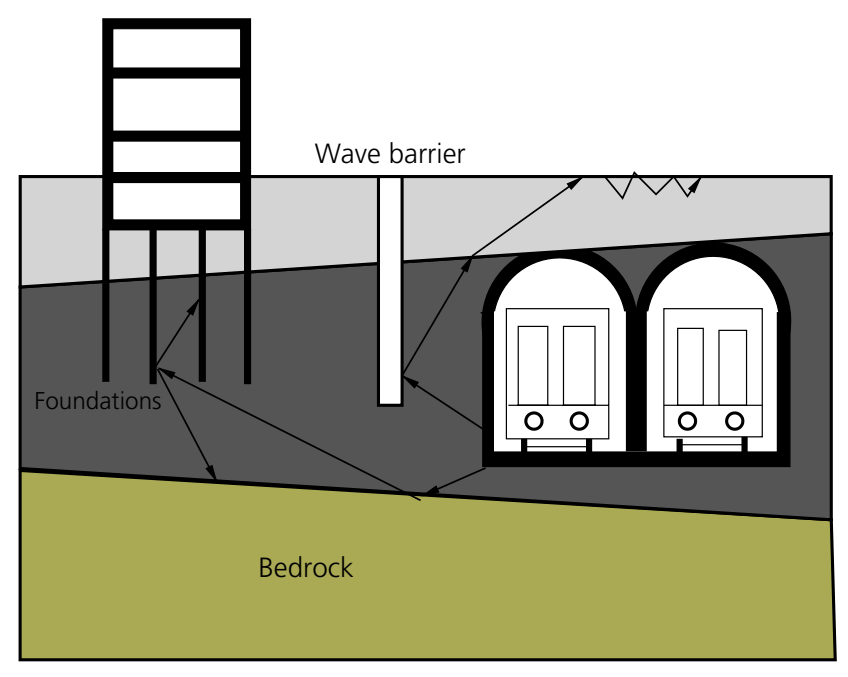

Figure 1. Configuration and idealisation of a soil-structure interaction problem

the effect of wave barriers of various shapes and embedded mats is examined. Moreover, the flexibility of the unstructured mesh grids is used to investigate train-induced surface vibration in the presence of conventional railway embankments or embankment with vertical walls.

The irregular domain is handled with FEM by discretising it into three-node triangular finite elements with two degrees of freedom at each node, horizontal and vertical displacements. The effect of the two lateral semi-infinite media is replaced by the nodal forces they apply on the irregular domain. Following Waas (1972), the appropriate governing differential equation of motion is applied for each individual sub-layer. Free vibration of all sub-layers, while ensuring compatibility of stresses and displacements at the interfaces, as well as taking into account the free and fixed boundary conditions, leads to two global second-order eigenvalue problems, one for each lateral boundary. For example, the eigenvalue problem related to the right lateral boundary has the form

$$
\text { 1. }\left[k^{2} \mathbf{A}+i k \mathbf{B}+\mathbf{G}-\omega^{2} \mathbf{M}\right] \boldsymbol{u}_{\mathrm{R}}=0
$$

where $k$ is the wavenumber, $\omega$ is the circular frequency of the load, $i$ is the pure imaginary number such that $i^{2}=-1$ and $\boldsymbol{u}_{\mathrm{R}}$ represents the eigenvectors of the vibrating right boundary. Matrices A, G and $\mathbf{M}$, which are symmetrical, are obtained from the geometry and material properties of the horizontal sub-layers of the right semi-infinite domain. Matrix B, which is a skew matrix, depends only on the material properties of the sub-layers. Each matrix is of dimension $2(n+1) \times 2(n+1)$, with $n$ being the number of sub-layers. These global matrices are obtained by assembling the corresponding sub-layer matrices $\mathbf{A}_{j}, \mathbf{B}_{j}, \mathbf{G}_{j}$ and $\mathbf{M}_{j}$, of dimensions $(4 \times 4)$. The solution 
of expression (1) yields to computing $2(n+1)$ eigenvalues and their corresponding eigenvectors. If the soil domain is overlying rigid bedrock, the last two rows and columns of the global matrices are omitted owing to base fixity. This yields to an eigenvalue problem with $2 n$ eigenvalues and corresponding eigenvectors. Hamdan et al. (2013) investigated the effect of selecting a few eigenmodes and hence reducing the computational cost of the method.

Kausel and Roësset (1981) derived an exact form for the dynamic impedance matrix for SV-P waves in a half-space. An approximate matrix was obtained by expanding the exact matrix using a Taylor series about $k=0$ and retaining the first three terms of the series (Hull and Kausel, 1984). The approximate matrix is expressed as

2.

$$
\begin{aligned}
\mathbf{K}_{\mathrm{app}}= & i \omega \rho c_{\mathrm{s}}\left[\begin{array}{cc}
1 & 0 \\
0 & 1 / \gamma
\end{array}\right]+\mu \frac{(1-2 \gamma)}{\gamma} k\left[\begin{array}{ll}
0 & 1 \\
1 & 0
\end{array}\right] \\
& +i \mu \frac{c_{\mathrm{s}}}{2 \omega} k^{2}\left[\begin{array}{cc}
-(2-\gamma) / \gamma & 0 \\
0 & (1-2 \gamma) / \gamma^{3}
\end{array}\right]
\end{aligned}
$$

where $\rho$ represents the half-space density, $\mu$ is the shear modulus of the half-space and $\gamma$ represents the ratio of the shear wave velocity $c_{\mathrm{s}}$ to the dilatational wave velocity $c_{\mathrm{p}}$ of the half-space. The approximate matrix of expression (2) is a second-order equation in the wave number $k$ and it relates the tractions and the displacements at the half-space surface. Hence, the half-space is taken into account using the inverse Fourier transform on the tractions-displacements to derive the stiffness matrix of the half-space elements and include the corner nodes. The soil-half-space interface is discretised into two-noded half-space elements. These elements share two nodes with the irregular region and thus the stiffness matrices of the half-space elements are assembled into the global stiffness matrix of expression (5). More details can be found in the references by Andrade (1999), Jones and Hunt (2011) and Hamdan (2013). The displacement field on each boundary is written as a combination of all computed eigenvalues and eigenvectors (Waas, 1972). The nodal forces vectors acting on the right and left boundaries of the model are expressed as

3. $\boldsymbol{P}_{\mathrm{R}}=-\mathbf{R} \boldsymbol{U}_{\mathrm{R}}$ and $\boldsymbol{P}_{\mathrm{L}}=-\mathbf{L} \boldsymbol{U}_{\mathrm{L}}$

The dynamic stiffness matrix of the consistent transmitting boundary of the right side regular region, $\mathbf{R}$, is expressed as

4. $\mathbf{R}=i \mathbf{A V H V} \mathbf{V}^{-1}+\mathbf{D}$

where $\mathbf{V}$ is the modal matrix containing all $2 n+1$ eigenvectors column-wise with the associated eigenvalues, with negative imaginary number, contained in the diagonal matrix $\mathbf{H}$.
Matrix D is a function of the horizontal sub-layers properties. The left side lateral region is treated in a similar way as was done for the right boundary, where the eigenvalues with positive imaginary part are used to form the diagonal matrix.

Usually, horizontal layers are used in the soil model and the left side boundary is a mirror of the right boundary. Hence, one eigenvalue problem can be solved. However, in this paper, two separate eigenvalue problems have been solved, as the problem includes an inclined base and non-horizontal soil layers. Therefore, the dynamic stiffness matrix of the left boundary is different from the one of the right boundary. The obtained matrices $\mathbf{R}$ and $\mathbf{L}$ are added to the stiffness matrix of the central region $\mathbf{K}$.

$$
\text { 5. }\left[(1+i \beta) \mathbf{K}_{\mathrm{s}}-\omega^{2} \mathbf{M}_{\mathrm{s}}+\mathbf{R}+\mathbf{L}\right] \boldsymbol{U}=\boldsymbol{P}
$$

where $\beta$ denotes the damping coefficient, and the global matrices $\mathbf{K}_{\mathrm{s}}$ and $\mathbf{M}_{\mathrm{s}}$ are obtained by assembling the stiffness and mass matrices of the three-node finite elements in the irregular soil domain and the two-node half-space finite elements. The external nodal vector is expressed by $\boldsymbol{P}$. A direct solver is used for the solution of the linear system (5) where the results are obtained as complex numbers for the vertical and horizontal displacements, for which the amplitudes $|v|$ and $|u|$ are computed, respectively.

\section{Theoretical and approximate solutions of the fundamental frequency of soil profiles}

The natural frequencies of a homogeneous soil layer of depth $H$ and subjected to a vertical harmonic load is given by expression (6). For more details see Kramer (1996).

$$
\text { 6. } f_{j}^{\mathrm{v}}=\frac{c_{\mathrm{p}}}{4 H}(2 j+1)
$$

where $f_{j}^{\mathrm{v}}$ represents the natural frequencies of the vertical loading and $j$ is an integer. The assumptions associated with deriving Equation 6 consider the soil layer to be isotropic linear and elastic, overlying horizontal rigid bedrock. Recently, Rovithis et al. (2011) investigated numerically and derived a closed-form solution for wave propagation in one dimensional (1D) layered inhomogeneous soil over rigid bedrock. The authors considered a case of an inhomogeneous soil layer over bedrock and an inhomogeneous soil layer overlying a homogeneous soil layer over horizontal bedrock. The fundamental frequency of a constant-depth soil layer could be estimated from expression (6); however, no such theoretical or even empirical solutions are available in the case of non-uniform depth of the soil. Therefore, numerical solutions could be an alternative and act as a guide for similar problems in practical engineering applications. For the two-layer soil profile case, Hadjian (2002) estimated the fundamental frequency by an 
approximate relationship involving the height of each soil layer, their fundamental periods and two parameters representing the ratio between the thicknesses of the soil layers.

7a.

$$
\begin{aligned}
& f=\frac{f_{1}}{\sqrt{\pi^{2} / 8\left\{0 \cdot 75+\left(f_{1} / f_{2}\right)^{2}\left[1+2\left(\rho_{1} H_{1} / \rho_{2} H_{2}\right)\right]\right\}}} \\
& \text { for } \frac{H_{1}}{H_{2}}>1
\end{aligned}
$$

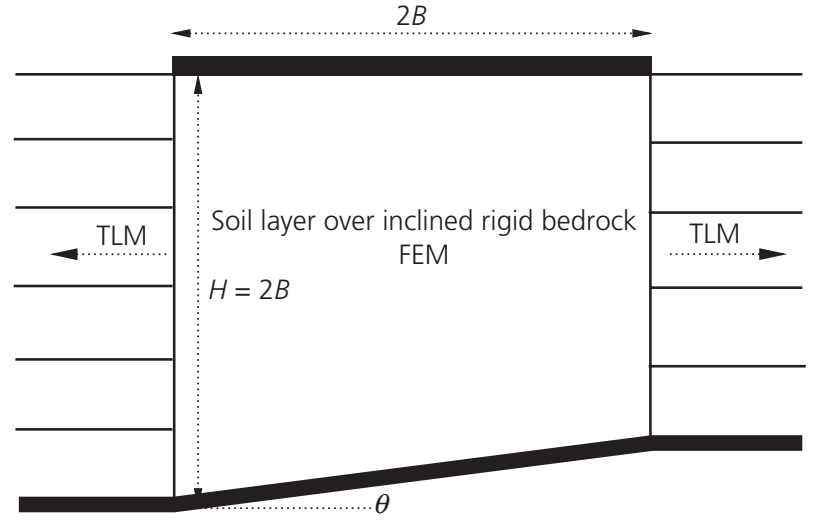

(a)

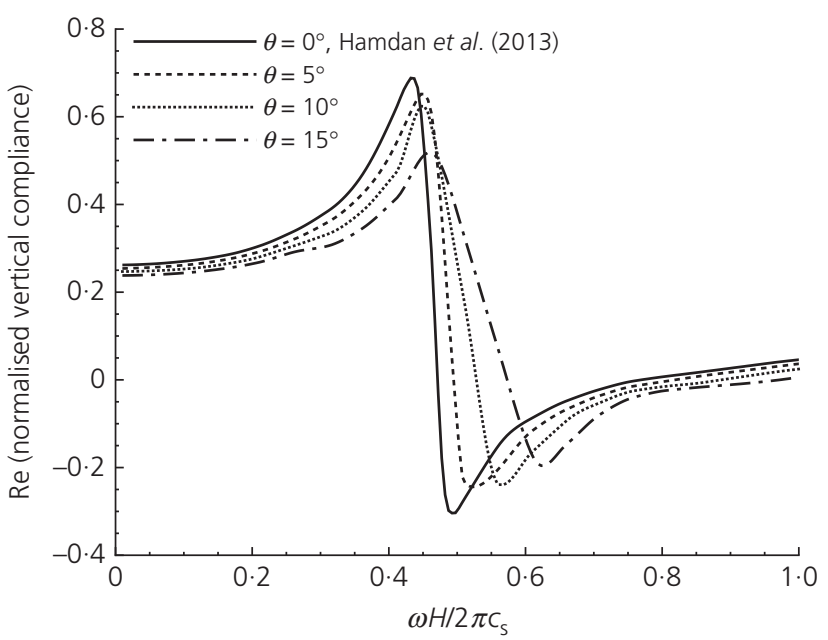

(b)

Figure 2. (a) Schematic diagram of a surface rigid foundation over soil layer over inclined base and (b) normalised real part of the compliance of the foundation (right image)

inclined rigid base. The domain is meshed using linear triangular elements with the mesh size not exceeding one-tenth of the Rayleigh wave length corresponding to the applied frequency. The centre of the foundation is subjected to a time-harmonic unit load with a range of frequencies $\omega$. The dynamic compliances of the foundation are computed and the real parts are plotted in Figure 2(b). As shown in the figure, the real parts of the vertical response of the foundation reach a peak around the dimensionless frequency of $0 \cdot 45$. In fact, this value is very close to the analytical value estimated from expression (6) to be around $0 \cdot 47$.

Next, the bedrock is inclined with angles of $5^{\circ}, 10^{\circ}$ and $15^{\circ}$. This is achieved by maintaining the thickness of the left lateral boundary constant at $H$ and changing the thickness of the right lateral boundary. This scenario leads to a decrease in the overall volume of the soil medium in the model. The dynamic response of the rigid foundation is examined by comparing 
its behaviour with the horizontal base case. As shown in Figure 2(b), for the angle of inclination of $5^{\circ}$ there is a shift or an increase in the fundamental frequency of the soil profile. This is more pronounced in the results of the vertical compliances. This is also observed for higher angles of inclination and, as the angle of inclination increases, the overall volume of the soil in the model decreases. As a consequence, the overall thickness of the soil model decreases and hence the denominator of expression (6) also decreases, leading the fundamental frequency to increase.

\subsection{Rigid surface foundation over a two-layer soil profile with inclined interface over horizontal bedrock}

The profile of a soil medium plays a key role in the dynamic response of embedded structures (Foinquinos and Roësset, 2000; Israil and Ahmad, 1989), where generally soil media are assumed to overlie a horizontal rigid base or a half-space. Chen (1994) simulated the effect of an inclined rigid base and inclined soil layers on the dynamic behaviour of surface foundations. The model consisted of three layers with the same angle of inclination. In this paper, a two-layer soil profile is dealt with to validate the presented model against the approximate solutions that are available for this case.

In this section, the soil profile is assumed to be underlain by a horizontal rigid base. The interface between the two layers is inclined in the same manner as in the previous section, leading to a decrease in the volume of the upper soil layer and an increase in the volume of the bottom layer. The total depth of the soil profile satisfies $(H / B=4)$, where $B$ is the half width of the foundation. The fundamental frequencies $f_{1}$ and $f_{2}$, of expressions (7a) and (7b), are related to the upper and the bottom layer, respectively. The relative densities, damping ratios and Poisson ratios of the two layers are all assumed to be equal to a unit, for simplicity. In the current analysis, the ratio $\left(H_{1} / H_{2}\right)$ is kept constant at 1 and the ratio of the relative stiffness between the two layers is chosen to be 2. $H_{1}$ and $H_{2}$ represent, respectively, the right lateral thicknesses of the upper and bottom soil layers. These will be varied according to the considered angles of inclination. Using the approximate solution of expression (7a), the fundamental frequency for the vertical loading can be evaluated. The dimensionless value is around $0 \cdot 17$, while the numerical code predicted a very close value of around $0 \cdot 15$. The same behaviour is obtained for the case of horizontal loading response. The interface between the soil layers is inclined with the angles $5^{\circ}, 10^{\circ}$ and $15^{\circ}$. It was shown by Hamdan (2013) that the inclination of the interface does not have a significant effect on the natural frequency of the soil profile. The variations of the compliances with the dimensionless frequency remain practically unchanged for all inclination cases considered in the analysis. This could be due to low angles of inclination or to the effect of the relative stiffness and thicknesses between the soil layers. This is investigated in the following sections.

\subsubsection{Effect of relative stiffness $\mu_{1} / \mu_{2}$}

Israil and Ahmad (1989) presented a parametric study using BEM for the dynamic behaviour of strip foundations overlying a homogeneous half-space, or a soil layer over a half-space and a soil layer over bedrock. The authors examined the effect of the material damping, the relative stiffness between the soil layer and the half-space and the effect of stratum depth considering only vertical loading. A similar parametric study is carried out in the current work to investigate the dynamic response of surface strip footings over two-layer soil media with inclined interface overlying horizontal bedrock. Here the effect of the relative stiffness of the two layers is studied.

Both layers have a Poisson ratio of 0.3 and a damping coefficient of $0 \cdot 05$. The ratio between their relative stiffness is taken as 0.5 and 4 . The normalised amplitudes of the vertical displacements are computed and plotted in Figure 3 for various angles of inclination of the interface between the two layers. From the results, it is clear that this has an insignificant effect on the vertical displacements under the centre of the foundation when the angle of inclination is changed. Various stiffness ratios were examined by Hamdan (2013) and the same behaviour was observed.

\subsubsection{Effect of relative thickness $H_{1} / H_{2}$}

The thicknesses of the two soil layers appear in the denominator of expressions (7a) and (7b), in the form of $\left(\rho_{1} H_{1}\right) /$ $\left(\rho_{2} \mathrm{H}_{2}\right)$, and each layer thickness is included, implicitly, in the terms expressing $f_{1}$ and $f_{1} / f_{2}$. Hence, they may have some effect on the fundamental frequency of the soil profile. This aspect is investigated here. For simplicity, Poisson ratio, damping ratio and soil densities are considered to be the same for both layers. The only difference in material properties is expressed in the ratio of the relative stiffness, $\mu_{1} / \mu_{2}$, which is taken as $0 \cdot 25$. The total depth of the stratum is kept constant at $4 B$. The ratio $H_{1} / H_{2}$ is varied between 0.25 and 4 and the results for ratios $0 \cdot 25$ and 4 are presented. The normalised amplitudes of the vertical displacements under the foundation are shown in Figure 4. The results show that the interface inclination has an influence on the dynamic compliances, especially at low values of $H_{1} / H_{2}$. For example, for $H_{1} / H_{2}=0 \cdot 25$, the normalised amplitudes of the displacements decrease when the angle of inclination $\theta$ increases. Moreover, the peak values show slight shifts with respect to the dimensionless frequency. As can be seen from Figure 4(a), the amplitudes of the vertical displacements, before and after the fundamental frequency, decrease when comparing to the case of the horizontal interface. This could be due to the relative stiffness of the soil layers. In other words, the bottom soil layer is stiffer than the upper layer. Hence, increasing the volume of the stiffer layer leads to decreasing the amplitudes of the vertical displacements. For the case of $H_{1} / H_{2}=4$, the interface inclination leads to very little change in comparison to the previous cases. A slight shift in the fundamental frequency is noticed. The amplitudes of the vertical displacements (above the fundamental 


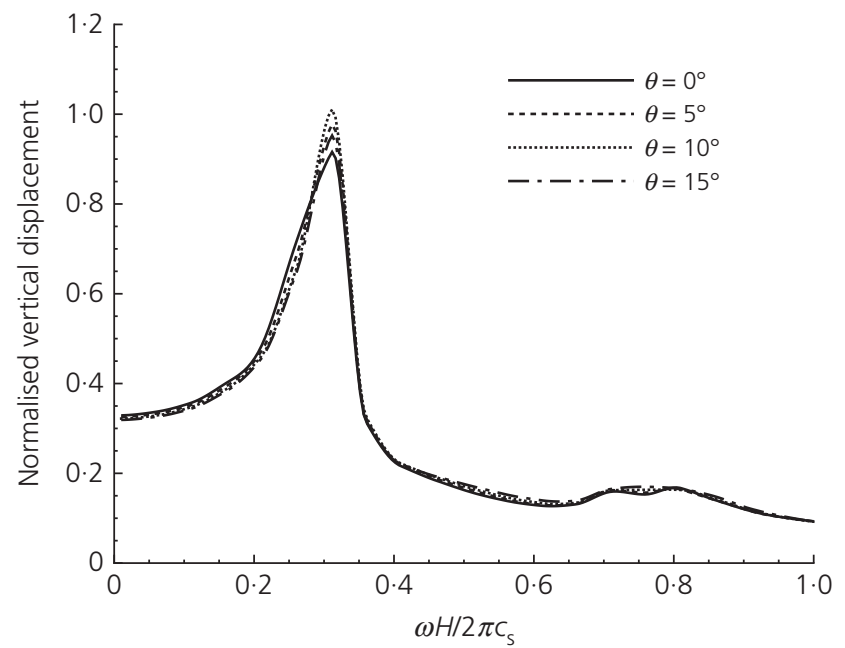

(a)

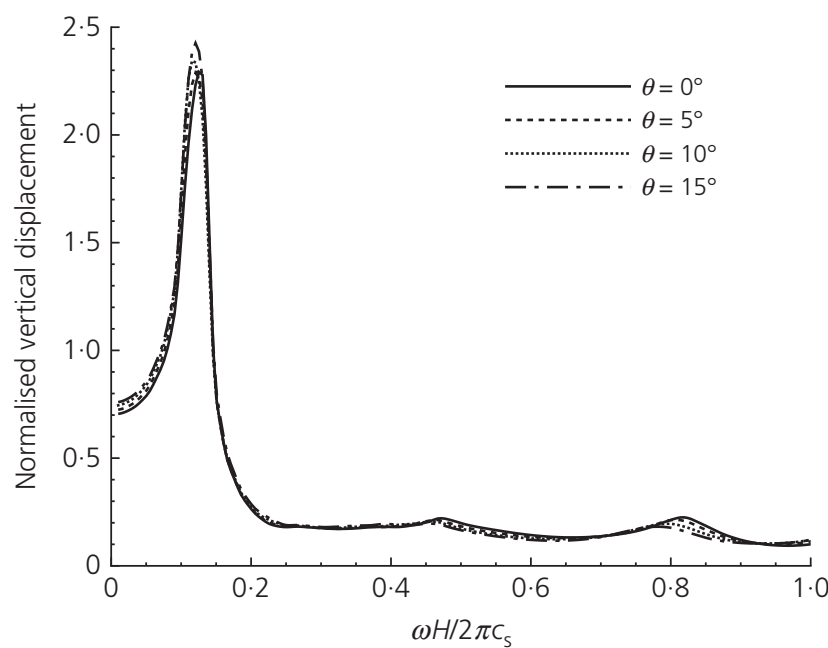

(b)

Figure 3. Normalised vertical displacement amplitudes under a surface rigid foundation overlaying a two-layer soil profile with non-horizontal interface: effect of relative stiffness: (a) $\mu_{1} / \mu_{2}=0 \cdot 5$; (b) $\mu_{1} / \mu_{2}=4$

frequency) have shown a slight increase compared to those of the horizontal layers. The same trend for relative thickness ratios of 1, 1.67 and 3 was observed (Hamdan, 2013).

\section{Dynamic behaviour of multi-layered soil profile}

The model is used in this section to simulate the dynamic behaviour of a multi-layered soil profile and compute its fundamental frequency. The computed fundamental frequencies, for varying parameters, are compared with those obtained using the expressions (7a) and (7b), where the successive use of a two-layer solution is adopted to compute the fundamental frequencies of the multi-layered medium. This is achieved first by computing the fundamental frequency of the first two layers,

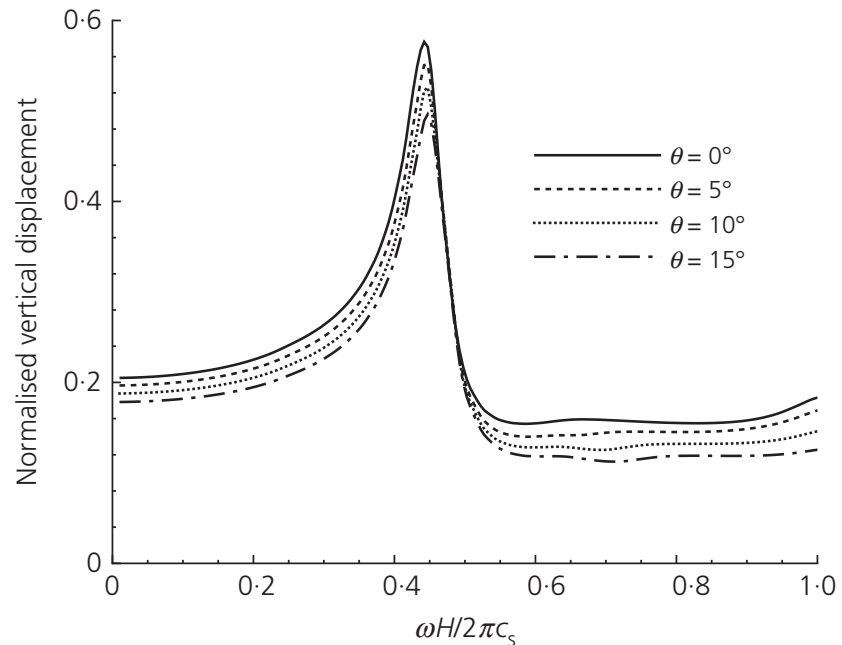

(a)

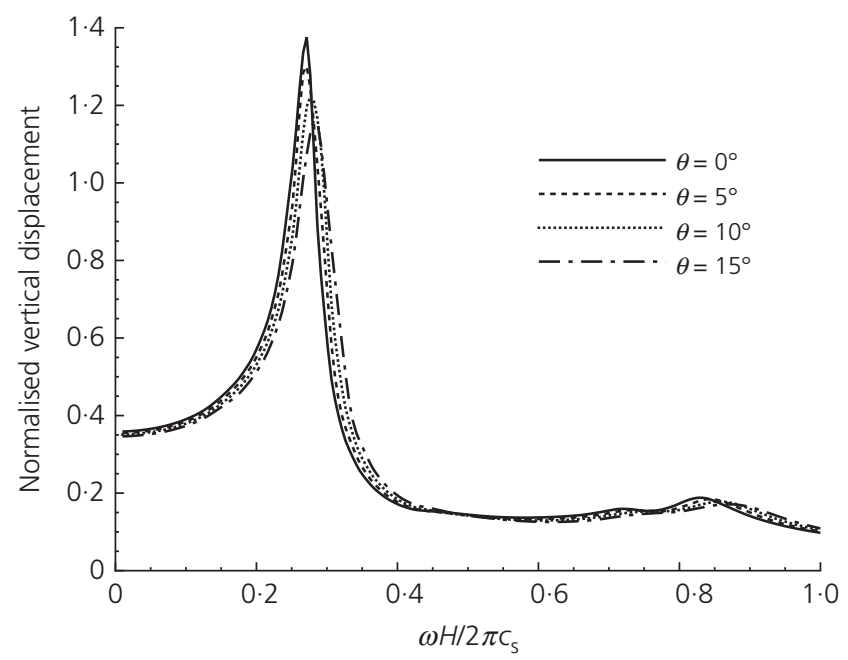

(b)

Figure 4. Normalised vertical displacement amplitudes under a surface rigid foundation overlaying a two-layer soil profile with non-horizontal interface: effect of relative thickness:

(a) $H_{1} / H_{2}=0.25$; (b) $H_{1} / H_{2}=4$

then the two layers are replaced by an equivalent layer and the fundamental frequency of the equivalent layer and the underlain layer is computed. The procedure is repeated until reaching the last soil layer. This is straightforward but it may lead to cumulating errors as it relies on successive use of approximations. Hence, a one-step numerical solution can tackle this problem more efficiently. Here, a soil profile with a linear increase in stiffness with depth, as in expression (8), is considered.

8. $\mu=\mu_{0}\left(1+\alpha z_{i}\right)$

where $\alpha$ is the rate of increase, $\mu_{0}$ is a reference shear modulus at the soil surface and $z_{i}$ is the depth below the soil surface. The soil density and Poisson ratio are assumed to be constant 
for convenience. Hence, the soil deposit is assumed to be soft at the surface and the stiffness is increased gradually to represent hard soil at the bottom. Table 1 shows soil properties corresponding to $\alpha=0 \cdot 5$.

A soil domain $30 \mathrm{~m}$ long and $10 \mathrm{~m}$ deep is used in this example. It is subjected to a vertical harmonic load of $1 \mathrm{kN}$ magnitude with a frequency varying from $0 \cdot 25 \mathrm{~Hz}$ to $15 \mathrm{~Hz}$. Three values of the rate of stiffness increase are used, $\alpha=0,0 \cdot 5$ and 1. The first case is equivalent to the homogeneous soil medium with the stiffness of the top layer.

The vertical displacement curves at the soil surface are computed for each loading frequency. The area under the displacement curve is computed using an integration scheme and plotted against the loading frequency. The results are shown in Figure 5. The numerical model estimated a fundamental frequency of $3.5 \mathrm{~Hz}$ for the case of an homogeneous profile, while expression (6) gives a value of $3.3 \mathrm{~Hz}$. Moreover, the current model predicted the fundamental frequencies of $5 \cdot 5 \mathrm{~Hz}$ and $7.5 \mathrm{~Hz}$, respectively, for $\alpha=0.5$ and $\alpha=1$, while $6 \mathrm{~Hz}$ and $7.9 \mathrm{~Hz}$ are obtained from expressions (7a) and (7b). It is clear that the numerically predicted fundamental frequencies and those obtained from the analytical and approximate solutions are in very good agreement. Hence, for more complex cases where analytical or approximate predictions are not possible, the presented numerical approach should become a very practical tool.

\section{Application to ground vibration reduction}

The developed coupled model is used in this work to model ground vibration reduction by an empty trench. This application is presented owing to the fact that the presence of discontinuities within the model produces multiple reflections and refractions. Thus, waves' amplitudes will vary within the homogeneous half-space. Consider a soil domain $20 \lambda_{\mathrm{R}}$ long and $4 \lambda_{\mathrm{R}}$ deep, as shown in Figure 6(a), subjected to a surface vertical

\begin{tabular}{llrll}
\hline$n$ & $\rho: \mathrm{kg} / \mathrm{m}^{3}$ & $c_{s}: \mathrm{m} / \mathrm{s}$ & $v$ & $\beta: \%$ \\
\hline 1 & 1550 & $70 \cdot 4$ & $0 \cdot 3$ & $2 \cdot 5$ \\
2 & 1550 & $80 \cdot 3$ & $0 \cdot 3$ & $2 \cdot 5$ \\
3 & 1550 & $89 \cdot 8$ & $0 \cdot 3$ & $2 \cdot 5$ \\
4 & 1550 & $98 \cdot 4$ & $0 \cdot 3$ & $2 \cdot 5$ \\
5 & 1550 & $105 \cdot 1$ & $0 \cdot 3$ & $2 \cdot 5$ \\
6 & 1550 & $113 \cdot 6$ & $0 \cdot 3$ & $2 \cdot 5$ \\
7 & 1550 & $120 \cdot 5$ & $0 \cdot 3$ & $2 \cdot 5$ \\
8 & 1550 & $127 \cdot 0$ & $0 \cdot 3$ & $2 \cdot 5$ \\
9 & 1550 & $133 \cdot 2$ & $0 \cdot 3$ & $2 \cdot 5$ \\
10 & 1550 & $139 \cdot 1$ & $0 \cdot 3$ & $2 \cdot 5$
\end{tabular}

Table 1. Layered soil profile properties for $\alpha=0.5$

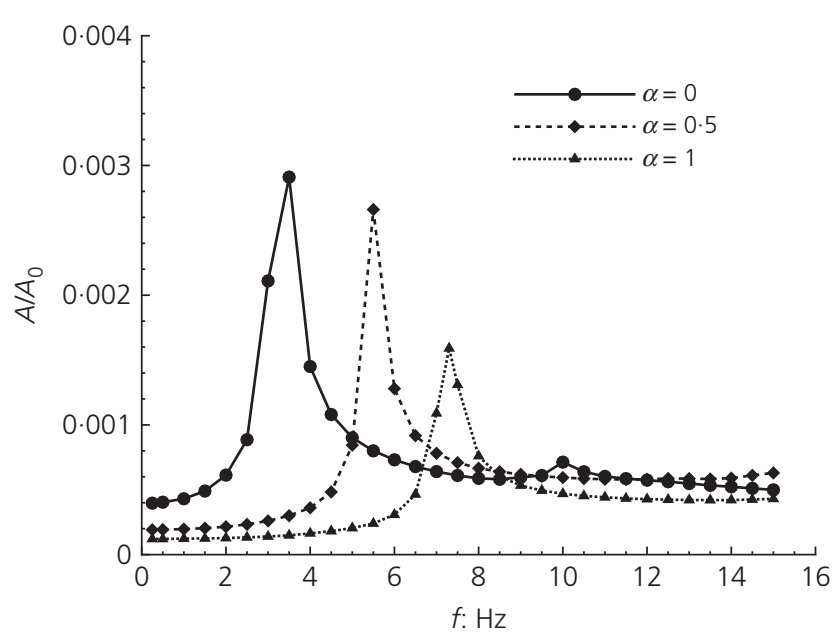

Figure 5. Fundamental frequency of a multi-layer soil profile over rigid base

harmonic load at the middle of the domain surface with a loading frequency of $31 \mathrm{~Hz}$. The derivation of the two-node half-space elements can be found in the references by Andrade (1999), Hamdan et al. (2015a) and Jones and Hunt (2011). It should be noted that the $4 \lambda_{\mathrm{R}}$ thickness of the model is very close to $0 \cdot 5 \lambda_{\mathrm{P}}$ as recommended by Hamdan et al. (2015a) for modelling halfspace media using this approach. Soil properties are adopted from the work of Yang and Hung (1997). An empty trench is placed at a distance of $5 \lambda_{\mathrm{R}}$ from the harmonic load. The vertical displacements at the surface of the domain are computed with and without the presence of the trench. The amplitude reduction factor, which relates the surface displacements with and without the trench, is computed for a distance of $5 \lambda_{\mathrm{R}}$ after the trench. Beskos et al. (1986) used a constant element-base boundary for investigating the screening effects of empty trenches, whereas Ahmad and Al-Hussaini (1991) employed an advanced direct BEM to deal with the same problem. As shown in Figure 6(b), the same trend is observed when comparing the coupled model results with the published results.

Another example is presented here to show the flexibility of adopting unstructured mesh grids to model problems of practical interest. The examined model consists of a harmonic load operating at the soil surface in the vicinity of a bridge. The model is $100 \mathrm{~m}$ long and $25 \mathrm{~m}$ deep, and is overlying rigid bedrock. The bridge has a $1 \mathrm{~m}$ wall thickness and $1 \mathrm{~m}$ of concrete thickness at its deck. This example is considered to show the diversity of cases which can be dealt with using the presented finite-element model. The loading can be applied on the bridge and levels of transmitted vibration can be monitored in the surrounding field. The distance between the applied load and the edge of the bridge, $X$, is varied from $5 \mathrm{~m}$ to $20 \mathrm{~m}$ and the area under the vertical displacement curves at the surface of the bridge is computed for a range of the loading frequency. Material properties used in the analysis are shown 


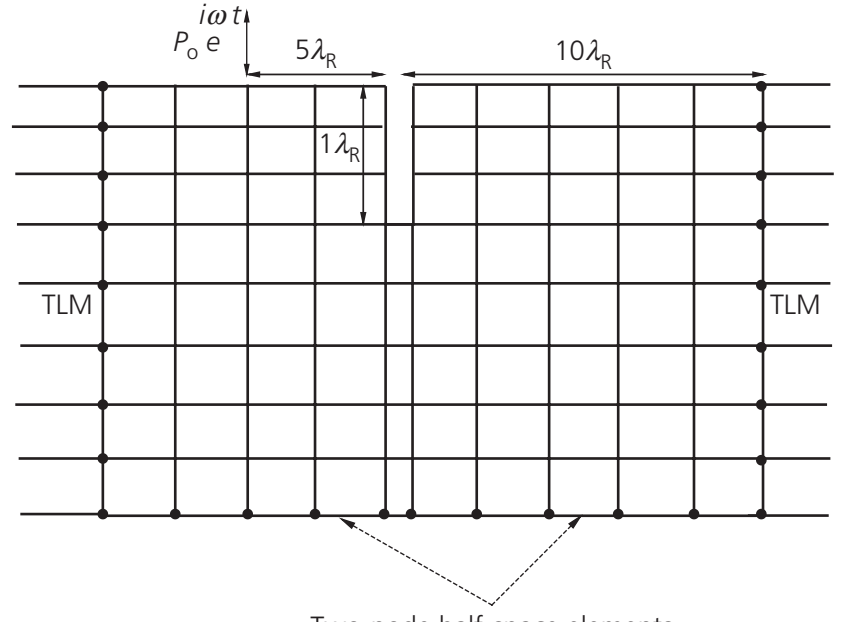

Two-node half-space elements

(a)

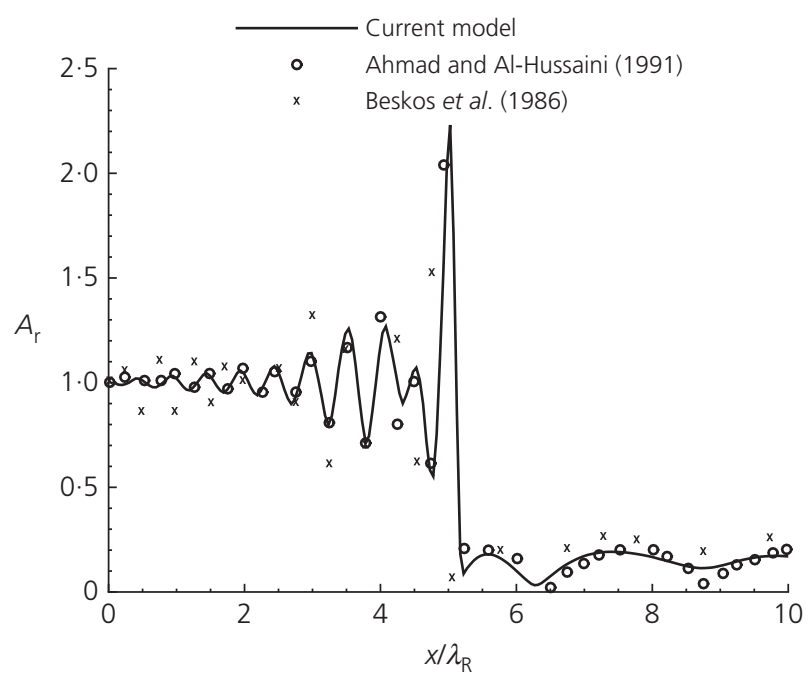

(b)

Figure 6. (a) Half-space finite-element model representation; (b) amplitude reduction factor of surface ground-borne vibration induced by a harmonic load on homogeneous half-space with distance from trench

in Table 2. First, the elasticity modulus of the soil, $E_{\mathrm{s}}$, is considered to be 0.0033 of the concrete, $E_{\mathrm{c}}$. For this case the first natural frequency of the examined model is around $2 \cdot 2 \mathrm{~Hz}$. The area under the vertical displacement curves for the ranges of frequencies is computed using the numerical model and plotted in Figure 7. At first, the load is assumed to be $5 \mathrm{~m}$ away from the left edge of the bridge. The previous figure shows that the bridge experiences displacement amplification at around $2 \mathrm{~Hz}$. This is in agreement with the computed value of the natural frequency using expression (6). The amplification decays beyond this frequency. The same response is obtained when the load is applied at $10 \mathrm{~m}$ and $20 \mathrm{~m}$ away from the edge of the bridge. The ratio of soil-concrete elasticity

\begin{tabular}{lcrcc}
\hline & $\rho: \mathrm{kg} / \mathrm{m}^{3}$ & $E: \mathrm{MPa}$ & $v$ & $\beta: \%$ \\
\hline Concrete & 2500 & 15000 & $0 \cdot 15$ & 1 \\
Soil $E_{\mathrm{s}} / E_{\mathrm{c}}=0.0033$ & 1700 & 50 & $0 \cdot 35$ & $2 \cdot 5$ \\
Soil $E_{\mathrm{S}} / E_{\mathrm{c}}=0.01$ & 1700 & 150 & 0.35 & $2 \cdot 5$
\end{tabular}

Table 2. Concrete and soil material properties

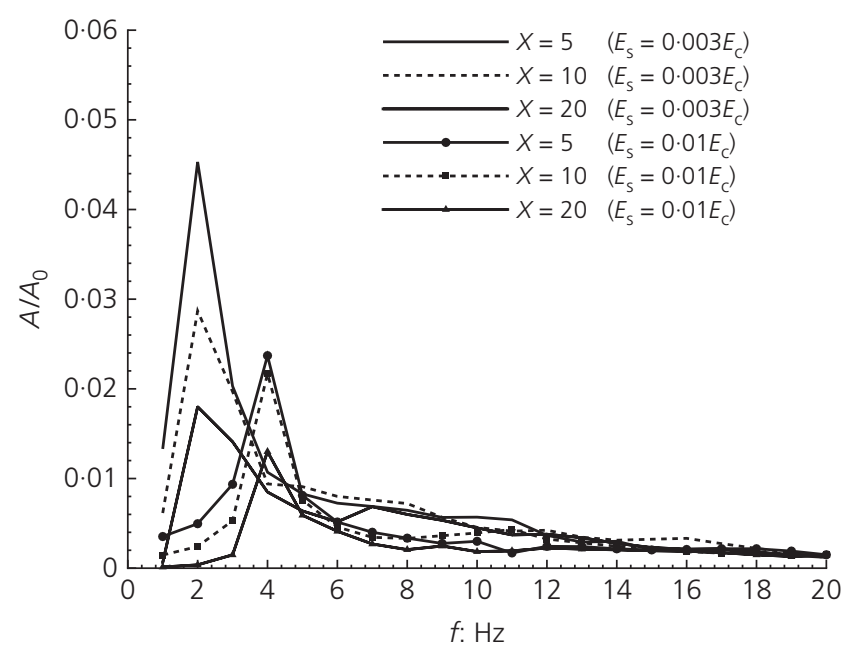

Figure 7. Area under vertical displacement curves along surface of bridge

moduli is increased to $0 \cdot 01$ and the response is computed for different locations of the applied load under various frequencies. The numerical model informs that the bridge amplification would occur at around $3.7 \mathrm{~Hz}$, which is in agreement with the value of $4 \mathrm{~Hz}$ computed using expression (6).

\section{Conclusions}

A frequency domain finite-element model is used in this work to model dynamic soil-structure interaction problems. The model combines the TLM and the PBC to simulate the semiinfinite extent of the soil. The model uses unstructured mesh grids to deal with problems of complex geometries with varying material properties.

Two types of applications are dealt with, namely, harmonic vibration of surface rigid foundation and harmonic-loadsinduced ground-borne vibration. The numerical model is employed to investigate the effect of non-horizontal soil profiles on the dynamic behaviour of surface rigid foundations.

Two examples of harmonic vibration of surface rigid foundations over bedrock are examined. The soil layer is assumed to overlie inclined rigid bedrock in the first model. The numerical results showed a slight increase in the fundamental frequency. 
This increase is affected by the angle of inclination. As the angle of inclination increases, the overall soil volume decreases and hence the fundamental frequency increases. A nonhorizontal layer interface is considered in the second example. The relative stiffness of the soil layers seems not to have any noticeable effect on the dynamic response of the soil medium. However, the relative thickness of the soil layers has a significant effect on the dynamic response of the soil medium, especially for a relative thickness ratio less than one. It is clear that the fundamental frequency has slightly shifted. For the case of $H_{1} / H_{2}=0 \cdot 25$, a slight reduction is observed in the fundamental frequency. More importantly, the amplitudes of the vertical displacements have decreased with increasing the angle of inclination for all applied dimensionless frequencies lower and greater than the cut-off frequency. This is due to decreasing the volume of the upper soil layer (less stiff layer). In the case of $H_{1} / H_{2}=4$, the upper soil layer is much deeper than the bottom layer and hence it has more influence on the fundamental frequency of the soil medium. This is clear as the amplitudes of displacements and the fundamental frequency are affected by the interface inclination.

The numerical model is also used to simulate the dynamic response of a multi-layered soil profile. The presented model predicted fundamental frequencies in good agreement with results of approximate solutions.

An example of surface ground vibration reduction is presented where an empty trench is placed between the load and the protected area. The model consisted of an homogeneous half-space meshed with structured elements. Another problem of practical interest is also presented where an unstructured mesh grid is employed, showing the flexibility and diversity of problems that can be dealt with using the presented numerical model.

\section{Declaration}

This work does not express the views of Lloyd's Register.

\section{REFERENCES}

Abascal R and Dominguez J (1984) Dynamic behaviour of strip footing on non-homogenous viscoelastic soils. Proceedings of the International Symposium on Dynamic Soil-Structure Interaction, Minneapolis, MN, USA, pp. 25-35.

Ahmad A and Al-Hussaini TM (1991) Simplified design for vibration screening by open and infilled trenches. Journal of Geotechnical Engineering 117(1): 67-88.

Andrade PW (1999) Implementation of Second-Order Absorbing Boundary Conditions in Frequency-Domain Computations. PhD thesis, University of Texas, Austin, TX, USA.

Barbosa JMO, Park J and Kausel E (2012) Perfectly matched layers in the thin layer method. Computer Methods in Applied Mechanics and Engineering 217-220: 262-274.
Barbosa J, Costa PA and Calçada R (2015) Abatement of railway induced vibrations: numerical comparison of trench solutions. Engineering Analysis with Boundary Elements 55: 122-139.

Basu U and Chopra AK (2003) Perfectly matched layers for time-harmonic elastodynamics of unbounded domains: theory and finite element implementation. Computer Methods in Applied Mechanics and Engineering 192(11-12): 1337-1375.

Basu U and Chopra AK (2004) Perfectly matched layers for transient elastodynamics of unbounded domains. International Journal for Numerical Methods in Engineering 59(8): 1039-1074.

Beskos DE, Dasgupta G and Vardoulakis IG (1986) Vibration isolation using open or filled trenches, part 1: 2-D homogeneous soil. Computational Mechanics 1(1): 43-63.

Chen HT (1994) Dynamic stiffness of strip footings on irregular soil stratum. Journal of the Chinese Institute of Engineers 17(3): 377-384.

Costa PA, Calçada R and Cardoso AS (2012) Track-ground vibrations induced by railway traffic: in-situ measurements and validations of a 2.5D FEM-BEM model. Soil Dynamics and Earthquake Engineering 32(1): 111-128.

Foinquinos R and Roësset JM (2000) Elastic layered half-spaces subjected to dynamic surface loads. In Wave Motion in Earthquake Engineering (Kausel E and Manolis GD (eds)). WIT Press, Southampton, UK, chapter 4, pp. 141-191.

Geuzaine C and Remacle JF (2009) Gmsh: A 3-D finite element mesh generator with built-in pre-and post-processing facilities. International Journal for Numerical Methods in Engineering 79(11): 1309-1331.

Hadjian AH (2002) Fundamental period and mode shape of layered soil profiles. Soil Dynamics and Earthquake Engineering 22(9-12): 885-891.

Hall WS and Oliveto G (2003) Boundary Element Methods for Soil-Structure Interaction. Kluwer Academic Publishers, Dordrecht, the Netherlands.

Hamdan N (2013) Numerical Modelling of Wave Propagation in Soil Media. PhD thesis, Heriot-Watt University, Edinburgh, UK.

Hamdan N, Laghrouche O, El Kacimi A and Woodward PK (2013) Consistent transmitting boundary conditions with a reduced number of eigenmodes for wave propagation in elastic media. Computers and Geotechnics 53: 9-16.

Hamdan N, Laghrouche O, El Kacimi A and Woodward PK (2015a) Combined paraxial-consistent boundary conditions finite element model for simulating wave propagation in elastic half-space media. Soil Dynamics and Earthquake Engineering 70: 80-92.

Hamdan N, Laghrouche O, Woodward PK and Mahmood MS (2015b) Ground vibration reduction analysis using a frequency-domain finite element approach. Construction and Building Materials 92: 95-103. 
Hull S and Kausel E (1984) Dynamic loads in layered half-spaces. Proceedings of the 5th Engineering Mechanics in Civil Engineering Division, Specialty Conference, Wyoming, USA, pp. 201-204.

Ikeda I Jr and Tassoulas JL (2008) Wave computations in irregular layered media using a semidiscrete finite element perturbation method. International Journal for Numerical Methods in Engineering 76(3): 376-399.

Israil ASM and Ahmad S (1989) Dynamic vertical compliance of strip foundations in layered soils. Earthquake Engineering and Structrual Dynamics 18(7): 933-950.

Jones S and Hunt H (2011) Effect of inclined soil layers on surface vibration from underground railways using the thin-layer method. Journal of Engineering Mechanics 137(12): 887-900.

Kausel E (1974) Forced Vibrations of Circular Foundations on Layered Media. Department of Civil Engineering, Massachusetts Institute of Technology, Cambridge, MA, USA, Research Report R74-11.

Kausel E and Roësset JM (1981) Stiffness matrices for layered soils. Bulletin of the Seismological Society of America 71(6): 1743-1761.

Kramer SL (1996) Geotechnical Earthquake Engineering. Prentice Hall, Upper Saddle River, NJ, USA.

Laghrouche O (1996) Numerical Simulation of Wave Propagation in Soil: Application to Vibration Isolation. $\mathrm{PhD}$ thesis, Université de Nantes, Nantes, France.

Lee JH, Kim JK and Tassoulas JL (2011a) Implementation of a second-order paraxial boundary condition for a water-saturated layered half-space in plane strain. Earthquake Engineering and Structural Dynamics 40(5): 531-550.

Lee JH, Kim JK and Tassoulas JL (2011b) Application of a second-order paraxial boundary condition to problems of dynamics of circular foundations on a porous layered halfspace. Soil Dynamics and Earthquake Engineering 31(3): 291-305.

Lee JH, Kim JK and Kim JH (2014) Nonlinear analysis of soil-structure interaction using perfectly matched discrete layers. Computers and Structures 142: 28-44.

Liu YJ, Mukherjee S, Nishimura N et al. (2011) Recent advances and emerging applications of the boundary element method. Applied Mechanics Review 64(3): 1-38.

Lopes P, Costa PA, Ferraz M, Calçada R and Cardoso AS (2014) Numerical modelling of vibrations induced by railway traffic in tunnels: from the source to the nearby buildings. Soil Dynamics and Earthquake Engineering 61-62: 269-285.

Park J and Kausel E (2004) Numerical dispersion in the thin-layer method. Computers and Structures 82(7-8): 607-625.

Park SH and Tassoulas JL (2002) Time-harmonic analysis of wave propagation in unbounded layered strata with zigzag boundaries. Journal of Engineering Mechanics 128(3): 359-368.
Romero A, Galvín P and Domínguez J (2013a) 3D non-linear time domain FEM-BEM approach to soil-structure interaction problems. Engineering Analysis with Boundary Elements 37(3): 503-512.

Romero A, Solís M, Domínguez J and Galvín P (2013b) Soil-structure interaction in resonant railway bridges. Soil Dynamics and Earthquake Engineerings 47: 108-116.

Rovithis EN, Parashakis H and Mylonakis GE (2011) 1D harmonic response of layered inhomogeneous soil: analytical investigation. Soil Dynamics and Earthquake Engineering 31(7): 879-890.

Semblat JF, Gandomzadeh A and Lenti L (2010) A simple numerical absorbing layer method in elastodynamics. Mecanique 338(1): 24-32.

Spyrakos CC and Beskos DE (1986) Dynamic response of rigid strip-foundations by a time domain boundary element method. International Journal for Numerical Methods in Engineering 23(8): 1547-1565.

Tassoulas JL (1981) Elements for the Numerical Analysis of Wave Motion in Layered Media. Department of Civil Engineering, Massachusetts Institute of Technology, Cambridge, MA, USA, Research Report R81-2.

von Estroff O and Schmid G (1984) Application of the boundary element method to the analysis of the vibration behaviour of strip foundation on a soil layer. Proceedings of the International Symposium on Dynamic Soil-Structure Interaction, Minneapolis, MN, USA, pp. 11-17.

Waas G (1972) Linear Two-Dimensional Analysis of Soil Dynamics Prolems in Semi-Infinite Layered Media. $\mathrm{PhD}$ thesis, University of California, Berkeley, CA, USA.

Yang YB and Hung HH (1997) A parametric study of wave barriers for reduction of train induced vibrations. International Journal for Numerical Methods in Engineering 40(20): 3729-3747.

Yang YB, Hung HH and Hsu LC (2007) Ground vibrations due to underground trains considering soil-tunnel interaction. Interaction and Multiscale Mechanics 1(1): 157-175.

\section{HOW CAN YOU CONTRIBUTE?}

To discuss this paper, please email up to 500 words to the editor at journals@ice.org.uk. Your contribution will be forwarded to the author(s) for a reply and, if considered appropriate by the editorial board, it will be published as discussion in a future issue of the journal.

Proceedings journals rely entirely on contributions from the civil engineering profession (and allied disciplines). Information about how to submit your paper online is available at www.icevirtuallibrary.com/page/authors, where you will also find detailed author guidelines. 